\title{
SOME INTERESTING CONGRUENCES FOR BALLOT NUMBERS
}

\section{NEŞE ÖMÜR AND SIBEL KOPARAL}

Received 30 April, 2014

Abstract. In this paper, we determine the sums $\sum_{k=0}^{n-1}\left(\begin{array}{c}2 k+d \\ k\end{array}\right) / x^{k}, \sum_{k=0}^{n-1} k\left(\begin{array}{c}2 k+d \\ k\end{array}\right) / x^{k}$ and some congruences can be obtained by using them. For example, for an odd prime $p \neq 5$,

$$
\sum_{k=0}^{(p-1) / 2}(-1)^{k} k\left(\begin{array}{c}
2 k+d \\
k
\end{array}\right) \equiv \frac{(-1)^{d}}{5}\left(\frac{5}{p}\right)\left(F_{d+1-\left(\frac{5}{p}\right)}-(d+1) L_{d+1-\left(\frac{5}{p}\right)}\right) \quad(\bmod p),
$$

and for an odd prime $p$,

$$
\sum_{k=0}^{(p-1) / 2} \frac{k}{(-4)^{k}} B(k, d) \equiv-(-2)^{d-1}\left(\frac{2}{p}\right) d P_{d+1-\left(\frac{2}{p}\right)} \quad(\bmod p),
$$

where $d \in\{0, \ldots,(p-1) / 2\}, F_{n}$ is the $n$th Fibonacci number, $L_{n}$ is the $n$th Lucas number and $P_{n}$ is the $n$th Pell number. $(\dot{\bar{p}})$ denotes the Legendre symbol.

2010 Mathematics Subject Classification: 11B65; 05A10; 11A07

Keywords: congruences, binomial coefficients, Ballot numbers

\section{INTRODUCTION}

The some elementary combinatorial properties of the Catalan and Ballot numbers are given in [2], [4] and [3]. In [1], [7], É. Lucas and N.J. Fine gave how to compute binomial coefficients modulo a prime. Lucas Theorem is given as follows:

If $p$ is a prime, $n, m, n_{0}$ and $m_{0}$ are non-negative integers, and $n_{0}, m_{0}$ are both less than $p$, then

$$
\left(\begin{array}{c}
n p+n_{0} \\
m p+m_{0}
\end{array}\right) \equiv\left(\begin{array}{c}
n \\
m
\end{array}\right)\left(\begin{array}{c}
n_{0} \\
m_{0}
\end{array}\right) \quad(\bmod p)
$$

The Catalan numbers are given by

$$
C_{n}=\frac{1}{n+1}\left(\begin{array}{c}
2 n \\
n
\end{array}\right)=\left(\begin{array}{c}
2 n \\
n
\end{array}\right)-\left(\begin{array}{c}
2 n \\
n+1
\end{array}\right), n \in \mathbb{N} .
$$


In [2], the Catalan numbers are special cases of the Ballot numbers

$$
B(n, k)=\frac{k}{2 n+k}\left(\begin{array}{c}
2 n+k \\
n
\end{array}\right) .
$$

In [13], Z.W. Sun and R. Tauraso obtained $\sum_{k=0}^{p^{a}-1}\left(\begin{array}{c}2 k \\ k+d\end{array}\right) / m^{k}$ and $\sum_{k=0}^{p-1}\left(\begin{array}{c}2 k \\ k+d\end{array}\right) / k m^{k-1}$ mod $p$ for all $d=0,1, \ldots, p^{a}$, where $m$ is any integer not divisible by $p$. For example, they showed that if $p \neq 2,5$, then

$$
\sum_{k=0}^{p-1}(-1)^{k}\left(\begin{array}{c}
2 k \\
k
\end{array}\right) \equiv-5 \frac{F_{p-\left(\frac{p}{5}\right)}}{p}(\bmod p) .
$$

In [8], Z.W. Sun determined $\sum_{k=0}^{p^{a}-1}\left(\begin{array}{c}2 k \\ k+d\end{array}\right) / m^{k} \bmod p^{2}$ for $d=0,1$; for example,

$$
\sum_{k=0}^{p^{a}-1}\left(\begin{array}{c}
2 k \\
k+d
\end{array}\right) / m^{k} \equiv\left(\frac{m^{2}-4 m}{p^{a}}\right)+\left(\frac{m^{2}-4 m}{p^{a-1}}\right) u_{p-\left(\frac{m^{2}-4 m}{p}\right)}\left(\bmod p^{2}\right),
$$

where an odd prime $p$ and $a, m \in \mathbb{Z}$ with $a>0, p \nmid m$.

In [11], Z.W. Sun used Lucas quotients in order to obtain $\sum_{k=0}^{(p-1) / 2}\left(\begin{array}{c}2 k \\ k\end{array}\right) / m^{k}$ modulo $p^{2}$ for any integer $m \neq \equiv 0 \quad(\bmod p)$; especially, he determined the following congruence:

$$
\sum_{k=0}^{(p-1) / 2} \frac{\left(\begin{array}{c}
2 k \\
k
\end{array}\right)}{16^{k}} \equiv\left(\frac{3}{p}\right) \quad\left(\bmod p^{2}\right)
$$

In [9], Z.W. Sun gave the following congruence:

$$
\sum_{k=0}^{(p-3) / 2} \frac{\left(\begin{array}{c}
2 k \\
k
\end{array}\right)}{(2 k+1) 4^{k}} \equiv(-1)^{(p+1) / 2} q_{p}(2) \quad\left(\bmod p^{2}\right),
$$

where an odd prime $p$ and $q_{p}(2)$ is a Fermat quotient.

In [6], S. Koparal and N. Ömür presented congruences involving central binomial coefficients and harmonic numbers. For example, for an odd prime $p$,

$$
\sum_{k=0}^{(p-1) / 2}(-1)^{k}\left(\begin{array}{c}
2 k \\
k
\end{array}\right) H_{k-1} \equiv \frac{2^{p}}{p}\left(2 F_{p+1}-5^{(p-1) / 2}-1\right) \quad(\bmod p),
$$

where $H_{n}$ is the $n$th harmonic number. 
In [5], K.H. Pilehrood et all gave that for a prime $p \neq 2,5$,

$$
\sum_{k=0}^{(p-3) / 2} \frac{\left(\begin{array}{c}
2 k \\
k
\end{array}\right) F_{2 k+1}}{(2 k+1) 16^{k}} \equiv(-1)^{(p+1) / 2} \frac{F_{p}-\left(\frac{p}{5}\right)}{p} \quad\left(\bmod p^{2}\right) .
$$

In this paper, we determine the sums $\sum_{k=0}^{n-1}\left(\begin{array}{c}2 k+d \\ k\end{array}\right) / x^{k}, \sum_{k=0}^{n-1} k\left(\begin{array}{c}2 k+d \\ k\end{array}\right) / x^{k}$ and some congruences can be obtained by using them.

Two sequences $\left\{u_{n}(x)\right\}$ and $\left\{v_{n}(x)\right\}$ of polynomials are defined by for $n>0$

$$
u_{n+1}(x)=x u_{n}(x)-u_{n-1}(x) \text { and } v_{n+1}(x)=x v_{n}(x)-v_{n-1}(x),
$$

where $u_{0}(x)=0, u_{1}(x)=1$ and $v_{0}(x)=2, v_{1}(x)=x$, respectively. The characteristic equation $y^{2}-x y+1=0$ of the sequences $\left\{u_{n}(x)\right\}$ and $\left\{v_{n}(x)\right\}$ has two roots

$$
\alpha(x)=\frac{x+\sqrt{\Delta}}{2} \text { and } \beta(x)=\frac{x-\sqrt{\Delta}}{2},
$$

where $\Delta=x^{2}-4$. The Binet formulas of the sequences $\left\{u_{n}(x)\right\}$ and $\left\{v_{n}(x)\right\}$ are as follows:

$$
u_{n}(x)=\frac{(\alpha(x))^{n}-(\beta(x))^{n}}{\alpha(x)-\beta(x)} \text { and } v_{n}(x)=(\alpha(x))^{n}+(\beta(x))^{n},
$$

respectively. Clearly, for any $n \in \mathbb{N}$,

$$
\begin{gathered}
x u_{n}(x)+v_{n}(x)=2 u_{n+1}(x), \\
u_{n}(x)+\frac{x+2}{\Delta} v_{n}(x)=\frac{2}{\Delta}\left(v_{n+1}(x)+v_{n}(x)\right), \\
\Delta u_{n}(x)=v_{n+1}(x)-v_{n-1}(x) .
\end{gathered}
$$

It is seen that

$$
\begin{aligned}
& (-1)^{n-1} u_{n}(-3)=F_{2 n} \text { and }(-1)^{n} v_{n}(-3)=L_{2 n}, \\
& (-1)^{n-1} u_{n}(-6)=\frac{1}{2} P_{2 n} \text { and }(-1)^{n} v_{n}(-6)=Q_{2 n},
\end{aligned}
$$

where $F_{n}$ and $L_{n}$ are the $n$th Fibonacci number and $n$th Lucas number, and $P_{n}$ and $Q_{n}$ are the $n$th Pell number and the $n$th Pell-Lucas number, respectively.

\section{SOME CONGRUENCES RELATED TO BALLOT NUMBERS}

In this section, we will investigate some congruences with the combinatorial identities. Now, we give the following lemmas for further use. 
Lemma 1. Let $r, s \in \mathbb{Z}$ and $D=r^{2}-4 s$. Suppose that $p$ is an odd prime with $p \nmid s D$. Then

$$
\left(\frac{r \pm \sqrt{D}}{2}\right)^{p-\left(\frac{D}{p}\right)} \equiv s^{\left(1-\left(\frac{D}{p}\right)\right) / 2} \quad(\bmod p)
$$

where $\frac{r \pm \sqrt{D}}{2}$ are roots of the equation $y^{2}-r y+s=0$ [10].

Lemma 2. For any $n \in \mathbb{N}$, we have

$$
\frac{d}{d x}\left(u_{n}(x-2)\right)=\frac{1}{\Delta}\left((n+1) v_{n}(x-2)-2 u_{n+1}(x-2)\right),
$$

where $\Delta$ as before.

Proof. By differentiating both sides of $\alpha(x-2)=\frac{x-2+\sqrt{\Delta}}{2}$, we write

$$
\frac{d}{d x}(\alpha(x-2))=\frac{x-2+\sqrt{\Delta}}{2 \sqrt{\Delta}}=\frac{\alpha(x-2)}{\sqrt{\Delta}} .
$$

Similarly, it is clearly seen that

$$
\frac{d}{d x}(\beta(x-2))=\frac{-\beta(x-2)}{\sqrt{\Delta}} \text { and } \frac{d}{d x}(\sqrt{\Delta})=\frac{2 \alpha(x-2)}{\sqrt{\Delta}}-1=\frac{2 \beta(x-2)}{\sqrt{\Delta}}+1 .
$$

Thus

$$
\begin{aligned}
\frac{d}{d x}\left(u_{n}(x-2)\right)= \\
=\frac{d}{d x}\left(\frac{\alpha^{n}(x-2)-\beta^{n}(x-2)}{\sqrt{\Delta}}\right) \\
=\frac{1}{\Delta}\left(\left(n \frac{\alpha^{n}(x-2)}{\sqrt{\Delta}}+n \frac{\beta^{n}(x-2)}{\sqrt{\Delta}}\right) \sqrt{\Delta}\right. \\
\left.\quad-\left(\frac{2 \alpha(x-2)}{\sqrt{\Delta}}-1\right) \alpha^{n}(x-2)+\left(\frac{2 \beta(x-2)}{\sqrt{\Delta}}+1\right) \beta^{n}(x-2)\right) \\
=\frac{1}{\Delta}\left(n \alpha^{n}(x-2)+n \beta^{n}(x-2)-2\left(\frac{\alpha^{n+1}(x-2)-\beta^{n+1}(x-2)}{\sqrt{\Delta}}\right)\right. \\
\left.+\alpha^{n}(x-2)+\beta^{n}(x-2)\right) \\
=\frac{1}{\Delta}\left(n v_{n}(x-2)-2 u_{n+1}(x-2)+v_{n}(x-2)\right) \\
=\frac{1}{\Delta}\left((n+1) v_{n}(x-2)-2 u_{n+1}(x-2)\right) .
\end{aligned}
$$

This concludes the proof. 
Theorem 1. For any $n, d \in \mathbb{Z}^{+}$, we have

$$
\begin{aligned}
\sum_{k=0}^{n-1}\left(\begin{array}{c}
2 k+d \\
k
\end{array}\right) x^{n-1-k}= & \sum_{k=0}^{n+d-1}\left(\begin{array}{c}
2 n+d \\
k
\end{array}\right) u_{n+d-k}(x-2) \\
& -x^{n+\lfloor d / 2\rfloor}\left(\varepsilon u_{\lfloor d / 2\rfloor+1}(x-2)+u_{\lfloor d / 2\rfloor}(x-2)\right),
\end{aligned}
$$

where $\varepsilon=\left(1-(-1)^{d}\right) / 2$.

Proof. To prove (2.2), we shall apply induction method on $n$.

For $n=1$, we must show that for $d \in \mathbb{Z}^{+}$,

$$
\begin{aligned}
& \sum_{k=0}^{d}\left(\begin{array}{c}
d+2 \\
k
\end{array}\right) u_{d+1-k}(x-2)= \\
& =1+x^{\lfloor d / 2\rfloor+1}\left(\varepsilon u_{\lfloor d / 2\rfloor+1}(x-2)+u_{\lfloor d / 2\rfloor}(x-2)\right) .
\end{aligned}
$$

We have

$$
\left.\begin{array}{l}
\sum_{k=0}^{d}\left(\begin{array}{c}
d+2 \\
k
\end{array}\right) u_{d+1-k}(x-2)= \\
=1+\sum_{k=0}^{d+2}\left(\begin{array}{c}
d+2 \\
k
\end{array}\right) u_{d+1-k}(x-2)=1+\sum_{k=0}^{d+2}\left(\begin{array}{c}
d+2 \\
k
\end{array}\right) u_{k-1}(x-2) \\
=1+\frac{1}{\alpha(x-2)-\beta(x-2)}\left\{\frac{1}{\alpha(x-2)} \sum_{k=0}^{d+2}\left(\begin{array}{c}
d+2 \\
k
\end{array}\right)(\alpha(x-2))^{k}\right. \\
-\frac{1}{\beta(x-2)} \sum_{k=0}^{d+2}\left(\begin{array}{c}
d+2 \\
k
\end{array}\right)(\beta(x-2))^{k}
\end{array}\right\}
$$

Using the identity $\alpha(x-2) \beta(x-2)=1$, we get

$$
\begin{aligned}
& \sum_{k=0}^{d}\left(\begin{array}{c}
d+2 \\
k
\end{array}\right) u_{d+1-k}(x-2)= \\
& =1+\frac{1}{\alpha(x-2)-\beta(x-2)}\left\{(\beta(x-2))(1+\alpha(x-2))^{d+2}\right. \\
& \left.\quad-(\alpha(x-2))(1+\beta(x-2))^{d+2}\right\} .
\end{aligned}
$$


From $d=\lfloor d / 2\rfloor+\lfloor d / 2\rfloor+\varepsilon$, we rewrite

$$
\begin{aligned}
& \sum_{k=0}^{d}\left(\begin{array}{c}
d+2 \\
k
\end{array}\right) u_{d+1-k}(x-2)= \\
& =1+\frac{1}{\alpha(x-2)-\beta(x-2)} \\
& \quad \times\left\{(\beta(x-2))(1+\alpha(x-2))^{\varepsilon}(1+\alpha(x-2))^{\lfloor d / 2\rfloor+1}(1+\alpha(x-2))^{\lfloor d / 2\rfloor+1}\right. \\
& \left.\quad-(\alpha(x-2))(1+\beta(x-2))^{\varepsilon}(1+\beta(x-2))^{\lfloor d / 2\rfloor+1}(1+\beta(x-2))^{\lfloor d / 2\rfloor+1}\right\} .
\end{aligned}
$$

Using the identity $(1+\alpha(x-2))(1+\beta(x-2))=x$, we get

$$
\begin{aligned}
\sum_{k=0}^{d}\left(\begin{array}{c}
d+2 \\
k
\end{array}\right) u_{d+1-k}(x-2)= \\
=1+\frac{1}{\alpha(x-2)-\beta(x-2)} \\
\quad \times\left\{(\beta(x-2)+\varepsilon)(1+\alpha(x-2))^{\lfloor d / 2\rfloor+1}(1+\alpha(x-2))^{\lfloor d / 2\rfloor+1}\right. \\
\left.\quad-(\alpha(x-2)+\varepsilon)(1+\beta(x-2))^{\lfloor d / 2\rfloor+1}(1+\beta(x-2))^{\lfloor d / 2\rfloor+1}\right\} \\
=1+\frac{x^{\lfloor d / 2\rfloor+1}}{\alpha(x-2)-\beta(x-2)}\left\{(\alpha(x-2))^{\lfloor d / 2\rfloor}-(\beta(x-2))^{\lfloor d / 2\rfloor}\right. \\
\left.\quad+\varepsilon(\alpha(x-2))^{\lfloor d / 2\rfloor+1}-\varepsilon(\beta(x-2))^{\lfloor d / 2\rfloor+1}\right\} \\
=1+x^{\lfloor d / 2\rfloor+1}\left(u_{\lfloor d / 2\rfloor}(x-2)+\varepsilon u_{\lfloor d / 2\rfloor+1}(x-2)\right) .
\end{aligned}
$$

So, (2.2) holds for $n=1$.

We assume that the result is true for some integer $n \geq 1$.

We must show that for $n+1,(2.2)$ holds. By the induction hypothesis, for any $d \in \mathbb{Z}^{+}$, we write

$$
\begin{aligned}
& \sum_{k=0}^{n}\left(\begin{array}{c}
2 k+d \\
k
\end{array}\right) x^{n-k}= \\
& =\left(\begin{array}{c}
2 n+d \\
n
\end{array}\right)+x \sum_{k=0}^{n-1}\left(\begin{array}{c}
2 k+d \\
k
\end{array}\right) x^{n-1-k} \\
& =\left(\begin{array}{c}
2 n+d \\
n
\end{array}\right)+x \sum_{k=0}^{n+d-1}\left(\begin{array}{c}
2 n+d \\
k
\end{array}\right) u_{n+d-k}(x-2) \\
& -x^{n+1+\lfloor d / 2\rfloor}\left(\varepsilon u_{\lfloor d / 2\rfloor+1}(x-2)+u_{\lfloor d / 2\rfloor}(x-2)\right)
\end{aligned}
$$




$$
\begin{aligned}
= & \left(\begin{array}{c}
2 n+d \\
n
\end{array}\right)+x \sum_{k=0}^{n+d-1}\left(\begin{array}{c}
2 n+d \\
k
\end{array}\right) u_{n+d-k}(x-2) \\
& -2 \sum_{k=0}^{n+d-1}\left(\begin{array}{c}
2 n+d \\
k
\end{array}\right) u_{n+d-k}(x-2)+2 \sum_{k=0}^{n+d-1}\left(\begin{array}{c}
2 n+d \\
k
\end{array}\right) u_{n+d-k}(x-2) \\
& -\sum_{k=0}^{n+d-1}\left(\begin{array}{c}
2 n+d \\
k
\end{array}\right) u_{n+d-1-k}(x-2)+\sum_{k=0}^{n+d-1}\left(\begin{array}{c}
2 n+d \\
k
\end{array}\right) u_{n+d-1-k}(x-2) \\
& -x^{n+\lfloor d / 2\rfloor+1}\left(\varepsilon u_{\lfloor d / 2\rfloor+1}(x-2)+u_{\lfloor d / 2\rfloor}(x-2)\right) \\
= & (x-2) \sum_{k=0}^{n+d}\left(\begin{array}{c}
2 n+d \\
k
\end{array}\right) u_{n+d-k}(x-2)-\sum_{k=0}^{n+d}\left(\begin{array}{c}
2 n+d \\
k
\end{array}\right) u_{n+d-1-k}(x-2) \\
& +2 \sum_{k=0}^{n+d-1}\left(\begin{array}{c}
2 n+d \\
k
\end{array}\right) u_{n+d-k}(x-2)+\sum_{k=0}^{n+d-1}\left(\begin{array}{c}
2 n+d \\
k
\end{array}\right) u_{n+d-1-k}(x-2) \\
& -x^{n+\lfloor d / 2\rfloor+1}\left(\varepsilon u_{\lfloor d / 2\rfloor+1}(x-2)+u_{\lfloor d / 2\rfloor}(x-2)\right) .
\end{aligned}
$$

Since $(x-2) u_{n}(x-2)=u_{n+1}(x-2)+u_{n-1}(x-2)$, we have

$$
\begin{aligned}
& \sum_{k=0}^{n}\left(\begin{array}{c}
2 k+d \\
k
\end{array}\right) x^{n-k}= \\
& =\sum_{k=0}^{n+d}\left(\begin{array}{c}
2 n+d \\
k
\end{array}\right) u_{n+d+1-k}(x-2)+2 \sum_{k=-1}^{n+d-1}\left(\begin{array}{c}
2 n+d \\
k
\end{array}\right) u_{n+d-k}(x-2) \\
& +\sum_{k=-2}^{n+d-1}\left(\begin{array}{c}
2 n+d \\
k
\end{array}\right) u_{n+d-1-k}(x-2) \\
& -x^{n+\lfloor d / 2\rfloor+1}\left(\varepsilon u_{\lfloor d / 2\rfloor+1}(x-2)+u_{\lfloor d / 2\rfloor}(x-2)\right) \\
& =\sum_{k=0}^{n+d}\left(\begin{array}{c}
2 n+d \\
k
\end{array}\right) u_{n+d+1-k}(x-2)+2 \sum_{k=0}^{n+d}\left(\begin{array}{c}
2 n+d \\
k-1
\end{array}\right) u_{n+d+1-k}(x-2) \\
& +\sum_{k=0}^{n+d+1}\left(\begin{array}{c}
2 n+d \\
k-2
\end{array}\right) u_{n+d+1-k}(x-2) \\
& -x^{n+\lfloor d / 2\rfloor+1}\left(\varepsilon u_{\lfloor d / 2\rfloor+1}(x-2)+u_{\lfloor d / 2\rfloor}(x-2)\right) \text {, }
\end{aligned}
$$


and hence

$$
\begin{aligned}
& \sum_{k=0}^{n}\left(\begin{array}{c}
2 k+d \\
k
\end{array}\right) x^{n-k}= \\
& =\sum_{k=0}^{n+d}\left(\left(\begin{array}{c}
2 n+d \\
k
\end{array}\right)+2\left(\begin{array}{c}
2 n+d \\
k-1
\end{array}\right)+\left(\begin{array}{c}
2 n+d \\
k-2
\end{array}\right)\right) u_{n+d+1-k}(x-2) \\
& \quad-x^{n+\lfloor d / 2\rfloor+1}\left(\varepsilon u_{\lfloor d / 2\rfloor+1}(x-2)+u_{\lfloor d / 2\rfloor}(x-2)\right) .
\end{aligned}
$$

By the binomial identity $\left(\begin{array}{c}2 n+d \\ k\end{array}\right)+2\left(\begin{array}{c}2 n+d \\ k-1\end{array}\right)+\left(\begin{array}{c}2 n+d \\ k-2\end{array}\right)=\left(\begin{array}{c}2 n+d+2 \\ k\end{array}\right)$, we get

$$
\begin{aligned}
\sum_{k=0}^{n}\left(\begin{array}{c}
2 k+d \\
k
\end{array}\right) x^{n-k} & =\sum_{k=0}^{n+d}\left(\begin{array}{c}
2(n+1)+d \\
k
\end{array}\right) u_{n+d+1-k}(x-2) \\
& -x^{n+\lfloor d / 2\rfloor+1}\left(\varepsilon u_{\lfloor d / 2\rfloor+1}(x-2)+u_{\lfloor d / 2\rfloor}(x-2)\right) .
\end{aligned}
$$

Hence the result is true for all integers $n \geq 0$.

As a result of Theorem 1, we may give the following congruence.

Corollary 1. Let $p$ be an odd prime. Then

$$
\begin{aligned}
& \sum_{k=0}^{(p-1) / 2} \frac{1}{m^{k}}\left(\begin{array}{c}
2 k+d \\
k
\end{array}\right) \equiv m^{\lfloor d / 2\rfloor-(p-1) / 2} \\
& \quad \times\left\{(1-\varepsilon) u_{(p-1) / 2+\lfloor d / 2\rfloor}(m-2)+((m-1) \varepsilon+1) u_{(p+1) / 2+\lfloor d / 2\rfloor}(m-2)\right\} \\
& \quad-m^{\lfloor d / 2\rfloor+1}\left(\varepsilon u_{\lfloor d / 2\rfloor+1}(m-2)+u_{\lfloor d / 2\rfloor}(m-2)\right) \quad(\bmod p)
\end{aligned}
$$

where $\varepsilon, \Delta$ as before, $d \in\{0,1, \ldots,(p-1) / 2\}$ and $m \in \mathbb{Z}$ with $p \nmid m \Delta$.

Proof. Substituting $n=(p+1) / 2$ and $x=m$ in (2.2), we write

$$
\begin{aligned}
& \sum_{k=0}^{(p-1) / 2}\left(\begin{array}{c}
2 k+d \\
k
\end{array}\right) m^{(p-1) / 2-k}=\sum_{k=0}^{(p-1) / 2+d}\left(\begin{array}{c}
p+d+1 \\
k
\end{array}\right) u_{(p+1) / 2+d-k}(m-2) \\
& -m^{(p+1) / 2+\lfloor d / 2\rfloor}\left(\varepsilon u_{\lfloor d / 2\rfloor+1}(m-2)+u_{\lfloor d / 2\rfloor}(m-2)\right) .
\end{aligned}
$$

By the congruence in (1.1), it is easily seen that

$$
\begin{aligned}
& m^{(p-1) / 2} \sum_{k=0}^{(p-1) / 2} \frac{\left(\begin{array}{c}
2 k+d \\
k
\end{array}\right)}{m^{k}} \equiv \\
& \equiv \sum_{k=0}^{d+1}\left(\begin{array}{c}
d+1 \\
k
\end{array}\right) u_{(p+1) / 2+d-k}(m-2) \\
& \quad-m^{\lfloor d / 2\rfloor+(p+1) / 2}\left(\varepsilon u_{\lfloor d / 2\rfloor+1}(m-2)+u_{\lfloor d / 2\rfloor}(m-2)\right)
\end{aligned}
$$




$$
\begin{aligned}
= & \sum_{k=0}^{d+1}\left(\begin{array}{c}
d+1 \\
k
\end{array}\right) u_{(p-1) / 2+k}(m-2) \\
& -m^{\lfloor d / 2\rfloor+(p+1) / 2}\left(\varepsilon u_{\lfloor d / 2\rfloor+1}(m-2)+u_{\lfloor d / 2\rfloor}(m-2)\right) \\
= & \frac{(\alpha(m-2))^{(p-1) / 2}}{\alpha(m-2)-\beta(m-2)} \sum_{k=0}^{d+1}\left(\begin{array}{c}
d+1 \\
k
\end{array}\right)(\alpha(m-2))^{k} \\
& -\frac{(\beta(m-2))^{(p-1) / 2}}{\alpha(m-2)-\beta(m-2)} \sum_{k=0}^{d+1}\left(\begin{array}{c}
d+1 \\
k
\end{array}\right)(\beta(m-2))^{k} \\
& -m^{(p+1) / 2+\lfloor d / 2\rfloor}\left(\varepsilon u_{\lfloor d / 2\rfloor+1}(m-2)+u_{\lfloor d / 2\rfloor}(m-2)\right) \quad(\bmod p) .
\end{aligned}
$$

By Binomial theorem, we have

$$
\begin{aligned}
& m^{(p-1) / 2} \sum_{k=0}^{(p-1) / 2} \frac{\left(\begin{array}{c}
2 k+d \\
k
\end{array}\right)}{m^{k}} \equiv \\
& \equiv \frac{(\alpha(m-2))^{(p-1) / 2}(1+\alpha(m-2))^{d+1}}{\alpha(m-2)-\beta(m-2)}-\frac{(\beta(m-2))^{(p-1) / 2}(1+\beta(m-2))^{d+1}}{\alpha(m-2)-\beta(m-2)} \\
& -m^{(p+1) / 2+\lfloor d / 2\rfloor}\left(\varepsilon u_{\lfloor d / 2\rfloor+1}(m-2)+u_{\lfloor d / 2\rfloor}(m-2)\right) \quad(\bmod p) .
\end{aligned}
$$

From $d=\lfloor d / 2\rfloor+\lfloor d / 2\rfloor+\varepsilon$, then

$$
\begin{aligned}
& \sum_{k=0}^{(p-1) / 2} \frac{\left(\begin{array}{c}
2 k+d \\
k
\end{array}\right)}{m^{k}} \equiv \\
\equiv & \frac{1}{\alpha(m-2)-\beta(m-2)} \times \\
& \times\left\{(\alpha(m-2))^{(p-1) / 2}(1+\alpha(m-2))^{\lfloor d / 2\rfloor+\varepsilon+1}(1+\alpha(m-2))^{\lfloor d / 2\rfloor}\right. \\
& \left.-(\beta(m-2))^{(p-1) / 2}(1+\beta(m-2))^{\lfloor d / 2\rfloor+\varepsilon+1}(1+\beta(m-2))^{\lfloor d / 2\rfloor}\right\} \\
& -m^{(p+1) / 2+\lfloor d / 2\rfloor}\left(\varepsilon u_{\lfloor d / 2\rfloor+1}(m-2)+u_{\lfloor d / 2\rfloor}(m-2)\right) \quad(\bmod p) .
\end{aligned}
$$

Using the equalities of $\alpha(m-2) \beta(m-2)=1$ and $(1+\alpha(m-2))(1+\beta(m-2))=$ $m$, we have the proof.

For example, when $d=0$ and $m \in \mathbb{Z}$ with $p \nmid m$

$$
\sum_{k=0}^{(p-1) / 2} \frac{\left(\begin{array}{c}
2 k \\
k
\end{array}\right)}{m^{k}} \equiv\left(\frac{m(m-4)}{p}\right) \quad(\bmod p)[12]
$$


and

$$
\sum_{k=0}^{(p-1) / 2} \frac{1}{(-4)^{k}}\left(\begin{array}{c}
2 k+d \\
k
\end{array}\right) \equiv(-2)^{d}\left(\frac{2}{p}\right) P_{d-\left(\frac{2}{p}\right)}(\bmod p),
$$

where $d \in\{0,1, \ldots,(p-1) / 2\}$.

Theorem 2. For any $n, d \in \mathbb{Z}^{+}$, we have

$$
\begin{aligned}
& \sum_{k=0}^{n-1} k\left(\begin{array}{c}
2 k+d \\
k
\end{array}\right) x^{n-1-k}=\sum_{k=0}^{n+d-1}\left(\begin{array}{c}
2 n+d \\
k
\end{array}\right)\left((n-1) u_{n+d-k}(x-2)\right. \\
& \left.\quad-\frac{x}{\Delta}\left((n+d+1-k) v_{n+d-k}(x-2)-2 u_{n+d+1-k}(x-2)\right)\right) \\
& \quad+(\lfloor d / 2\rfloor+1) x^{n+\lfloor d / 2\rfloor}\left(\varepsilon u_{\lfloor d / 2\rfloor+1}(x-2)+u_{\lfloor d / 2\rfloor}(x-2)\right) \\
& \quad+\frac{x^{n+\lfloor d / 2\rfloor+1}}{\Delta}\left(\varepsilon\left((\lfloor d / 2\rfloor+2) v_{\lfloor d / 2\rfloor+1}(x-2)-2 u_{\lfloor d / 2\rfloor+2}(x-2)\right)\right. \\
& \left.\quad+(\lfloor d / 2\rfloor+1) v_{\lfloor d / 2\rfloor}(x-2)-2 u_{\lfloor d / 2\rfloor+1}(x-2)\right),
\end{aligned}
$$

where $\varepsilon, \Delta$ as before.

Proof. To prove equality (2.5), we take the derivative of (2.2) with respect to x:

$$
\begin{aligned}
& \sum_{k=0}^{n-1}\left(\begin{array}{c}
2 k+d \\
k
\end{array}\right)(n-1-k) x^{n-2-k}= \\
& =\sum_{k=0}^{n+d-1}\left(\begin{array}{c}
2 n+d \\
k
\end{array}\right) \frac{1}{\Delta}\left((n+d+1-k) v_{n+d-k}(x-2)-2 u_{n+d+1-k}(x-2)\right) \\
& \quad-(n+\lfloor d / 2\rfloor) x^{n+\lfloor d / 2\rfloor-1}\left(\varepsilon u_{\lfloor d / 2\rfloor+1}(x-2)+u_{\lfloor d / 2\rfloor}(x-2)\right) \\
& \quad-\frac{x^{n+\lfloor d / 2\rfloor}}{\Delta}\left(\varepsilon\left((\lfloor d / 2\rfloor+2) v_{\lfloor d / 2\rfloor+1}(x-2)-2 u_{\lfloor d / 2\rfloor+2}(x-2)\right)\right. \\
& \left.\quad+(\lfloor d / 2\rfloor+1) v_{\lfloor d / 2\rfloor}(x-2)-2 u_{\lfloor d / 2\rfloor+1}(x-2)\right),
\end{aligned}
$$

and then

$$
\begin{aligned}
& \sum_{k=0}^{n-1} k\left(\begin{array}{c}
2 k+d \\
k
\end{array}\right) x^{n-2-k}= \\
& =\frac{n-1}{x} \sum_{k=0}^{n-1}\left(\begin{array}{c}
2 k+d \\
k
\end{array}\right) x^{n-1-k} \\
& \quad-\frac{1}{\Delta} \sum_{k=0}^{n+d-1}\left(\begin{array}{c}
2 n+d \\
k
\end{array}\right)\left((n+d+1-k) v_{n+d-k}(x-2)-2 u_{n+d+1-k}(x-2)\right)
\end{aligned}
$$




$$
\begin{aligned}
& +(n+\lfloor d / 2\rfloor) x^{n+\lfloor d / 2\rfloor-1}\left(\varepsilon u_{\lfloor d / 2\rfloor+1}(x-2)+u_{\lfloor d / 2\rfloor}(x-2)\right) \\
& +\frac{x^{n+\lfloor d / 2\rfloor}}{\Delta}\left(\varepsilon\left((\lfloor d / 2\rfloor+2) v_{\lfloor d / 2\rfloor+1}(x-2)-2 u_{\lfloor d / 2\rfloor+2}(x-2)\right)\right. \\
& \left.(\lfloor d / 2\rfloor+1) v_{\lfloor d / 2\rfloor}(x-2)-2 u_{\lfloor d / 2\rfloor+1}(x-2)\right) .
\end{aligned}
$$

Multiplying both sides of (2.6) with $x$ and using Lemma 1, the proof is the complete.

Now, from Theorem 2, we have the following congruence:

Corollary 2. Let $p$ be an odd prime. Then

$$
\begin{aligned}
& \sum_{k=0}^{(p-1) / 2} k \frac{\left(\begin{array}{c}
2 k+d \\
k
\end{array}\right)}{m^{k}} \equiv \\
\equiv & -\frac{m^{\lfloor d / 2\rfloor+1-(p-1) / 2}}{\Delta} \times \\
& \times\left((d+2)\left(v_{(p+1) / 2+\lfloor d / 2\rfloor}(m-2)+\varepsilon v_{(p+1) / 2+\lfloor d / 2\rfloor+1}(m-2)\right)\right. \\
& \left.-2\left((1-\varepsilon) u_{(p+1) / 2+\lfloor d / 2\rfloor}(m-2)+((m-1) \varepsilon+1) u_{(p+1) / 2+\lfloor d / 2\rfloor+1}(m-2)\right)\right) \\
& +\frac{m^{\lfloor d / 2\rfloor+\varepsilon+1}}{\Delta}\left(2(\lfloor d / 2\rfloor+1)\left(v_{\lfloor d / 2\rfloor+1}(m-2)+(1-\varepsilon) v_{\lfloor d / 2\rfloor}(m-2)\right)\right. \\
& \left.-(2-\varepsilon) m u_{\lfloor d / 2\rfloor+1}(m-2)\right) \quad(\bmod p),
\end{aligned}
$$

where $\varepsilon, \Delta$ as before, $d \in\{0,1, \ldots,(p-1) / 2\}$ and $m \in \mathbb{Z}$ with $p \nmid m \Delta$.

Proof. Substituting $n=(p+1) / 2$ and $x=m$ in (2.5), we write

$$
\begin{aligned}
& \sum_{k=0}^{(p-1) / 2} k\left(\begin{array}{c}
2 k+d \\
k
\end{array}\right) m^{(p-1) / 2-k}= \\
= & \sum_{k=0}^{(p-1) / 2+d}\left(\begin{array}{c}
p+d+1 \\
k
\end{array}\right)\left(\frac{p-1}{2} u_{(p+1) / 2+d-k}(m-2)\right. \\
& \left.-\frac{m}{\Delta}\left(\left(\frac{p+1}{2}+d+1-k\right) v_{(p+1) / 2+d-k}(m-2)-2 u_{(p+1) / 2+d+1-k}(m-2)\right)\right) \\
& +(\lfloor d / 2\rfloor+1) m^{(p+1) / 2+\lfloor d / 2\rfloor}\left(\varepsilon u_{\lfloor d / 2\rfloor+1}(m-2)+u_{\lfloor d / 2\rfloor}(m-2)\right) \\
& +\frac{m^{(p+1) / 2+\lfloor d / 2\rfloor+1}}{\Delta}\left(\varepsilon\left((\lfloor d / 2\rfloor+2) v_{\lfloor d / 2\rfloor+1}(m-2)-2 u_{\lfloor d / 2\rfloor+2}(m-2)\right)\right. \\
& \left.+(\lfloor d / 2\rfloor+1) v_{\lfloor d / 2\rfloor}(m-2)-2 u_{\lfloor d / 2\rfloor+1}(m-2)\right) .
\end{aligned}
$$


By the congruence in (1.1), it is easily seen that

$$
\begin{aligned}
& m^{(p-1) / 2} \sum_{k=0}^{(p-1) / 2} \frac{k\left(\begin{array}{c}
2 k+d \\
k
\end{array}\right)}{m^{k}} \equiv \\
& \equiv \sum_{k=0}^{d+1}\left(\begin{array}{c}
d+1 \\
k
\end{array}\right)\left(-\frac{1}{2} u_{(p+1) / 2+d-k}(m-2)\right. \\
&\left.\quad-\frac{m}{\Delta}\left(\left(\frac{3}{2}+d-k\right) v_{(p+1) / 2+d-k}(m-2)-2 u_{(p+1) / 2+d+1-k}(m-2)\right)\right) \\
&+ \\
&+(\lfloor d / 2\rfloor+1) m^{(p+1) / 2+\lfloor d / 2\rfloor}\left(\varepsilon u_{\lfloor d / 2\rfloor+1}(m-2)+u_{\lfloor d / 2\rfloor}(m-2)\right) \\
&+\frac{m^{(p+1) / 2+\lfloor d / 2\rfloor+1}}{\Delta}\left(\varepsilon\left((\lfloor d / 2\rfloor+2) v_{\lfloor d / 2\rfloor+1}(m-2)-2 u_{\lfloor d / 2\rfloor+2}(m-2)\right)\right. \\
&\left.+(\lfloor d / 2\rfloor+1) v_{\lfloor d / 2\rfloor}(m-2)-2 u_{\lfloor d / 2\rfloor+1}(m-2)\right) \\
&=-\frac{1}{2} \sum_{k=0}^{d+1}\left(\begin{array}{c}
d+1 \\
k
\end{array}\right) u_{(p-1) / 2+k}(m-2)+\frac{2 m}{\Delta} \sum_{k=0}^{d+1}\left(\begin{array}{c}
d+1 \\
k
\end{array}\right) u_{(p+1) / 2+k}(m-2) \\
& \quad-\frac{m}{\Delta} \sum_{k=0}^{d+1}\left(\begin{array}{c}
d+1 \\
k
\end{array}\right)\left(k+\frac{1}{2}\right) v_{(p-1) / 2+k}(m-2) \\
&+(\lfloor d / 2\rfloor+1) m^{(p+1) / 2+\lfloor d / 2\rfloor}\left(\varepsilon u_{\lfloor d / 2\rfloor+1}(m-2)+u_{\lfloor d / 2\rfloor}(m-2)\right) \\
&+\frac{m^{(p+1) / 2+\lfloor d / 2\rfloor+1}}{\Delta}\left(\varepsilon\left((\lfloor d / 2\rfloor+2) v_{\lfloor d / 2\rfloor+1}(m-2)-2 u_{\lfloor d / 2\rfloor+2}(m-2)\right)\right. \\
&\left.+(\lfloor d / 2\rfloor+1) v_{\lfloor d / 2\rfloor}(m-2)-2 u_{\lfloor d / 2\rfloor+1}(m-2)\right) \quad(\bmod p) .
\end{aligned}
$$

From the Binet formulae of the sequences $\left\{u_{n}(m-2)\right\}$ and $\left\{v_{n}(m-2)\right\}$ and Binomial theorem, we have

$$
\begin{aligned}
& m^{(p-1) / 2} \sum_{k=0}^{(p-1) / 2} \frac{k\left(\begin{array}{c}
2 k+d \\
k
\end{array}\right)}{m^{k}} \equiv \\
& \equiv-\frac{1}{2} m^{\lfloor d / 2\rfloor}\left((1-\varepsilon) u_{(p-1) / 2+\lfloor d / 2\rfloor}(m-2)+((m-1) \varepsilon+1) u_{(p+1) / 2+\lfloor d / 2\rfloor}(m-2)\right) \\
&+\frac{2}{\Delta} m^{\lfloor d / 2\rfloor+1}\left((1-\varepsilon) u_{(p+1) / 2+\lfloor d / 2\rfloor}(m-2)+((m-1) \varepsilon+1) u_{(p+1) / 2+\lfloor d / 2\rfloor+1}(m-2)\right) \\
&-\frac{m^{\lfloor d / 2\rfloor+1}}{\Delta}(d+1)\left(v_{(p+1) / 2+\lfloor d / 2\rfloor}(m-2)+\varepsilon v_{(p+1) / 2+\lfloor d / 2\rfloor+1}(m-2)\right) \\
&-\frac{m^{\lfloor d / 2\rfloor+1}}{2 \Delta}\left((1-\varepsilon) v_{(p-1) / 2+\lfloor d / 2\rfloor}(m-2)+((m-1) \varepsilon+1) v_{(p+1) / 2+\lfloor d / 2\rfloor}(m-2)\right) \\
&+(\lfloor d / 2\rfloor+1) m^{(p+1) / 2+\lfloor d / 2\rfloor}\left(\varepsilon u_{\lfloor d / 2\rfloor+1}(m-2)+u_{\lfloor d / 2\rfloor}(m-2)\right)
\end{aligned}
$$




$$
\begin{aligned}
& +\frac{m^{(p+1) / 2+\lfloor d / 2\rfloor+1}}{\Delta}\left(\varepsilon\left((\lfloor d / 2\rfloor+2) v_{\lfloor d / 2\rfloor+1}(m-2)-2 u_{\lfloor d / 2\rfloor+2}(m-2)\right)\right. \\
& \left.+(\lfloor d / 2\rfloor+1) v_{\lfloor d / 2\rfloor}(m-2)-2 u_{\lfloor d / 2\rfloor+1}(m-2)\right) \quad(\bmod p) .
\end{aligned}
$$

From (1.2), (1.3) and (1.4), we obtained the desired result.

For example, for an odd prime $p \neq 5$

$$
\begin{aligned}
& \sum_{k=0}^{(p-1) / 2}(-1)^{k} k\left(\begin{array}{c}
2 k+d \\
k
\end{array}\right) \equiv \\
& \equiv \frac{(-1)^{d}}{5}\left(\frac{5}{p}\right)\left(F_{d+1-\left(\frac{5}{p}\right)}-(d+1) L_{d+1-\left(\frac{5}{p}\right)}\right) \quad(\bmod p),
\end{aligned}
$$

and for an odd prime $p$,

$$
\begin{aligned}
& \sum_{k=0}^{(p-1) / 2} \frac{k}{(-4)^{k}}\left(\begin{array}{c}
2 k+d \\
k
\end{array}\right) \equiv \\
& \equiv(-2)^{d-2}\left(\frac{2}{p}\right)\left(P_{d+1-\left(\frac{2}{p}\right)}-\frac{d+1}{2} Q_{d+1-\left(\frac{2}{p}\right)}\right) \quad(\bmod p),
\end{aligned}
$$

where $d \in\{0,1, \ldots,(p-1) / 2\}$.

From Corollary 1 and Corollary 2, clearly the congruences are given as follows:

Corollary 3. Let $p$ be an odd prime and $d \in\{0,1,2, \ldots,(p-1) / 2\}$. For $m \in \mathbb{Z}$ with $p \nmid m \Delta$, then

$$
\begin{aligned}
& \sum_{k=0}^{(p-1) / 2} \frac{B(k, d)}{m^{k}} \equiv \\
& \equiv m^{\lfloor(d+1) / 2\rfloor-(p-1) / 2-\varepsilon}\left\{u_{(p-1) / 2+\lfloor(d+1) / 2\rfloor-\varepsilon}(m-2)-u_{(p+1) / 2+\lfloor(d+1) / 2\rfloor}(m-2)\right\} \\
& -m^{\lfloor(d+1) / 2\rfloor}\left(u_{\lfloor(d-1) / 2\rfloor}(m-2)-u_{\lfloor(d-1) / 2\rfloor+2-\varepsilon}(m-2)\right)-\delta_{0, d} \quad(\bmod p),
\end{aligned}
$$

and

$$
\begin{aligned}
& \sum_{k=0}^{(p-1) / 2} \frac{k}{m^{k}} B(k, d) \equiv \\
& \equiv \frac{m^{\lfloor(d+1) / 2\rfloor}}{\Delta} d\left\{m^{1-\varepsilon}\left(v_{\lfloor(d+1) / 2\rfloor-\varepsilon}(m-2)-v_{\lfloor(d+1) / 2\rfloor+1}(m-2)\right)\right. \\
& \left.\quad-m^{-(p-1) / 2}\left(v_{(p+1) / 2+\lfloor(d-1) / 2\rfloor}(m-2)-v_{(p+1) / 2+\lfloor(d+1) / 2\rfloor+1-\varepsilon}(m-2)\right)\right\} \quad(\bmod p),
\end{aligned}
$$

where $\varepsilon, \Delta$ as before and $\delta_{i, j}$ is the Kronecker delta. 

get

Proof. Using the binomial identities $\left(\begin{array}{l}n \\ k\end{array}\right)=\frac{n}{k}\left(\begin{array}{c}n-1 \\ k-1\end{array}\right)$ and $\left(\begin{array}{l}n \\ k\end{array}\right)=\left(\begin{array}{c}n-1 \\ k\end{array}\right)+\left(\begin{array}{c}n-1 \\ k-1\end{array}\right)$, we

$$
\sum_{k=0}^{(p-1) / 2} \frac{B(k, d)}{m^{k}}=\sum_{k=0}^{(p-1) / 2} \frac{1}{m^{k}} \frac{d}{2 k+d}\left(\begin{array}{c}
2 k+d \\
k
\end{array}\right)=\sum_{k=0}^{(p-1) / 2} \frac{1}{m^{k}} \frac{d}{k}\left(\begin{array}{c}
2 k+d-1 \\
k-1
\end{array}\right) .
$$

For $k, d \in \mathbb{Z}^{+}$, it is known that

$$
\left(\begin{array}{c}
2 k+d-1 \\
k
\end{array}\right)=\left(\begin{array}{c}
2 k+d-1 \\
k-1
\end{array}\right)+\frac{d}{k}\left(\begin{array}{c}
2 k+d-1 \\
k-1
\end{array}\right) .
$$

So, we have

$$
\begin{aligned}
& \sum_{k=0}^{(p-1) / 2} \frac{B(k, d)}{m^{k}} \\
= & \sum_{k=0}^{(p-1) / 2} \frac{1}{m^{k}}\left(\left(\begin{array}{c}
2 k+d-1 \\
k
\end{array}\right)-\left(\begin{array}{c}
2 k+d-1 \\
k-1
\end{array}\right)\right) \\
= & \sum_{k=0}^{(p-1) / 2} \frac{1}{m^{k}}\left(\begin{array}{c}
2 k+d-1 \\
k
\end{array}\right)-\sum_{k=0}^{(p-1) / 2} \frac{1}{m^{k}}\left(\begin{array}{c}
2 k+d-1 \\
k-1
\end{array}\right) \\
= & \sum_{k=0}^{(p-1) / 2} \frac{1}{m^{k}}\left(\begin{array}{c}
2 k+d-1 \\
k
\end{array}\right)-\sum_{k=-1}^{(p-3) / 2} \frac{1}{m^{k+1}}\left(\begin{array}{c}
2 k+d+1 \\
k
\end{array}\right) \\
= & \sum_{k=0}^{(p-1) / 2} \frac{1}{m^{k}}\left(\begin{array}{c}
2 k+d-1 \\
k
\end{array}\right)-\frac{1}{m} \sum_{k=0}^{(p-3) / 2} \frac{1}{m^{k}}\left(\begin{array}{c}
2 k+d+1 \\
k
\end{array}\right)-\left(\begin{array}{c}
d-1 \\
-1
\end{array}\right) \\
= & \sum_{k=0}^{(p-1) / 2} \frac{1}{m^{k}}\left(\begin{array}{c}
2 k+d-1 \\
k
\end{array}\right)-\frac{1}{m} \sum_{k=0}^{(p-1) / 2} \frac{1}{m^{k}}\left(\begin{array}{c}
2 k+d+1 \\
k
\end{array}\right)-\delta_{0, d} \\
& +\frac{1}{m^{(p+1) / 2}}\left(\begin{array}{c}
p+d \\
(p-1) / 2
\end{array}\right) .
\end{aligned}
$$

Since $\left(\begin{array}{c}p+d \\ (p-1) / 2\end{array}\right) \equiv 0 \quad(\bmod p)$ for $d \in\{0,1,2, \ldots,(p-1) / 2\}$, from (1.1), we get

$$
\begin{aligned}
& \sum_{k=0}^{(p-1) / 2} \frac{B(k, d)}{m^{k}} \equiv \\
\equiv & \sum_{k=0}^{(p-1) / 2} \frac{1}{m^{k}}\left(\begin{array}{c}
2 k+d-1 \\
k
\end{array}\right)-\frac{1}{m} \sum_{k=0}^{(p-1) / 2} \frac{1}{m^{k}}\left(\begin{array}{c}
2 k+d+1 \\
k
\end{array}\right)-\delta_{0, d} \quad(\bmod p) .
\end{aligned}
$$


So, taking $d-1$ and $d+1$ instead of $d$ in (2.4), respectively, this concludes the proof.

For example, for an odd prime $p \neq 5$

$$
\sum_{k=0}^{(p-1) / 2}(-1)^{k} B(k, d) \equiv(-1)^{d+1}\left(\frac{5}{p}\right) L_{d-\left(\frac{5}{p}\right)^{-}} \delta_{0, d} \quad(\bmod p),
$$

and for an odd prime $p$,

$$
\sum_{k=0}^{(p-1) / 2} \frac{k}{(-4)^{k}} B(k, d) \equiv-(-2)^{d-1}\left(\frac{2}{p}\right) d P_{d+1-\left(\frac{2}{p}\right)}(\bmod p),
$$

where $d \in\{0,1,2, \ldots,(p-1) / 2\}$.

\section{REFERENCES}

[1] N. Fine, "Binomial coefficients modulo a prime," Amer. Math. Monthly, vol. 54, pp. 589-592, 1947.

[2] I. R. Gessel, "Super ballot numbers," J. Symbolic Computation, vol. 14, pp. 179-194, 1992.

[3] P. Hilton and J. Pedersen, "The ballot problem and catalan numbers," Nieuw Arch. Wisk., vol. 8, pp. 209-216, 1990.

[4] P. Hilton and J. Pedersen, "Catalan numbers, their generalization, and their uses," The Mathematical Intelligencer, vol. 13, pp. 64-75, 1991.

[5] T. P. K.H. Pilehrood and R. Tauraso, "Congruences concerning jacobi polynomials and apèry-like formulae," Int. J. of Number Theory, vol. 8, pp. 1789-1811, 2012.

[6] S. Koparal and N. Ömür, "On congruences related to central binomial coefficients, harmonic and lucas numbers," Turkish J. Math., vol. 40, pp. 973-985, 2016.

[7] E. Lucas, "Sur les congruences des nombres eulériens et des coefficients différentiels des fonctions trigonométriques, suivant un module premier," Bull. Soc. Math. France, vol. 6, pp. 49-54, $1877-$ 1878.

[8] Z. Sun, "Binomial coefficients, catalan numbers and lucas quotients," Sci. China Math., vol. 53, pp. 2473-2488, 2010.

[9] Z. Sun, "On congruences related to central binomial coefficients," J. Number Theory, vol. 131, pp. 2219-2238, 2011.

[10] Z. Sun, "On harmonic numbers and lucas sequences," Publ. Math Debrecen, vol. 80, pp. 1-17, 2012.

[11] Z. Sun, "Fibonacci numbers modulo cubes of primes," Taiwanese J. Math., vol. 17, pp. 15231543, 2013.

[12] Z. Sun, "Congruences involving generalized central trinomial coefficients," Sci. China Math., vol. 57, pp. 1375-1400, 2014.

[13] Z. Sun and R. Tauraso, "New congruences for central binomial coefficients," Adv. in Appl. Math., vol. 4, pp. 125-148, 2010.

Authors' addresses

Neșe Ömür

Kocaeli University, Mathematics Department, 41380 Kocaeli, Turkey

E-mail address: neseomur@kocaeli.edu.tr 
Sibel Koparal

Kocaeli University, Mathematics Department, 41380 Kocaeli, Turkey

E-mail address: sibel.koparal@kocaeli.edu.tr 